\title{
Comparative Analysis of NATO Requirements for Laboratory Identification of Chemical Agents and Military Technical Means for Its Implementation
}

\author{
T. Rozsypal* and R. Zahradníček \\ NBC Defence Institute, University of Defence in Brno, Czech Republic \\ The manuscript was received on 12 November 2020 and was accepted \\ after revision for publication as technical information on 24 February 2021.
}

\begin{abstract}
:
Deployable chemical laboratories are considered a highly specific part of the Armed Forces of the Czech Republic, intended for Chemical, Biological, Radiological and Nuclear Defence in operations. Their professional activity is determined by a number of scientific and technical requirements, which are formulated by standards for sample identification. To achieve the required degree of credibility, it is particularly important to have specific technical capacities. This instrumentation is crucial for the implementation of laboratory analyzes. The article describes the state of chemical laboratories of the Chemical Corps in the context of standardized requirements and discusses some points of selected Alliance agreements which the Armed Forces of the Czech Republic have signed.
\end{abstract}

\section{Keywords:}

analysis, chemical warfare agent, chemical corps, chemical support, identification, deployable laboratory

\section{Introduction}

Specialized units for the implementation of Chemical, Biological, Radiological and Nuclear (CBRN) Defence in the Armed Forces of the Czech Republic (ACR) include deployable chemical laboratories (Deployable Chemical Analytical Laboratory, hereinafter "DLAB-CHEM"), comprised in the organizational structures of the CBRN Defence battalions. They are used for qualified sampling and analysis of chemical substances; in addition, they provide expert advice to the supported task force com-

\footnotetext{
* Corresponding author: University of Defence in Brno, NBC Defence Institute, Víta Nejedlého No. 1, CZ-682 01 Vyškov, Czech Republic. Phone: +420 973452 338, Fax: +420 9734523 30, E-mail: tomas.rozsypal@unob.cz.ORCID0000-0002-0872-0876.
} 
mander, participate in the assessment of the chemical weapons threat level in the area of operation, and facilitate documentation to make the right military decisions.

Their general tasks are set out in the national military regulation Vševojsk-2-14 [1] as follows: sampling according to national and international standards; performing laboratory analyzes and confirming identification of the anticipated chemical hazard; quality control of environmental components; inspection of infrastructure equipment for the possibility of leakage of industrial hazardous substances; providing conclusions to competent authorities for making operational decisions; distribution and preparation of samples for transport to reference laboratories.

Laboratories must meet the minimum capability requirements for the specified level of identification. To achieve them, it is especially important to have specific technical capacities. This instrumentation is the basic determinant for meeting the requirements for the implementation of the specified level of identification.

The aim of the article is to compare the state of chemical laboratories of the ACR Chemical Corps against standardized scientific and technical requirements and to discuss the questionable points of selected Alliance agreements which the ACR has signed.

\section{Standards Defining Minimum Requirements}

In relation to DLAB-CHEM, the Bi-SC Capability Codes and Capability Statements [2] establish a NATO requirement for sets of capabilities to identify chemical warfare agents (CWAs) and industrial chemicals up to the level of confirmed identification in accordance with STANAG 4632 [3]. At the same time, DLAB-CHEM should be able to perform daily in the range of six to eight samples. This also pertains to the ability to identify explosives.

The requirements for DLAB-CHEM according to the NATO Deployable Laboratory Concept are specified in STANAG 4632 [3] and STANAG 4701, or more precisely in AEP-66 (NATO Handbook for Sampling and Identification of Biological, Chemical, and Radiological Agents [SIBCRA]) [4]. The evaluation of laboratory readiness before operational deployment is performed in accordance with the standardization agreement STANAG 2520 or ATP-3.8.1 Volume III (CBRN Defense Standards for Education, Training and Evaluation) [5].

By ratifying the STANAG 4632 standard, nations agree to develop national CBRN capabilities in accordance with the standard and to accept the results and conclusions provided by laboratories built in accordance with the requirements of the standard. STANAG 4632 states only general requirements and in specific matters it refers to AEP-66, resp. its predecessors AEP-10 (SIBCA Handbook) and AEP-49 (SIRA Handbook). DLAB-CHEM, which is described in STANAG 4632, is intended for laboratory analysis of chemicals meeting the level of "confirmed identification". The analysis time of one sample should not exceed 4-6 hours. There is also a list of CWAs and toxins (Tab. 1), which forms the basic capability of DLAB-CHEM, and represents a list of substances that the laboratory should be able to identify at the level of confirmed identification.

From the above, it is clear that the basic laboratory method for analysis in a DLAB-CHEM is the gas chromatography/mass spectrometry (GC/MS) method. However, it should be added that to reach a complete and credible conclusion of the analysis, it is necessary to supplement the results with other procedures, such as preliminary detection methods, that will speed up the analysis process, as well as precise 
and traceable sample preparation. Modern instrumentation for their implementation is not fully available in established technical systems or it is not fully usable in terms of unquestionable output.

Tab. 1 List of chemical warfare agents and toxins that form a basic capability of DLAB-CHEM according to STANAG 4632 [3]

\begin{tabular}{|c|c|c|}
\hline Name of substance & Code designation & Method of identification \\
\hline \hline Sulphur mustard & H & GC/MS \\
\hline Sesqui mustard & Q & GC/MS \\
\hline Oxygen mustard & T & GC/MS \\
\hline Nitrogen mustard 1 & HN-1 & GC/MS \\
\hline Nitrogen mustard 2 & HN-2 & GC/MS \\
\hline Nitrogen mustard 3 & HN-3 & GC/MS \\
\hline Lewisit 1 & L1 & GC/MS \\
\hline Lewisit 2 & L2 & GC/MS \\
\hline Lewisit 3 & L3 & GC/MS \\
\hline Diisopropyl fluorophosphate & DFP & GC/MS \\
\hline Tabun & GA & GC/MS \\
\hline Sarin & GB & GC/MS \\
\hline Soman & GD & GC/MS \\
\hline Ethylsarin & GE & GC/MS \\
\hline Cyclosarin & GF & GC/MS \\
\hline Intermediate Volatility Agent & IVA & Manual test kit \\
\hline Amiton & VG & Manual test kit \\
\hline VX & VX & Manual test kit \\
\hline Ricin & & Manual test kit \\
\hline Saxitoxin & & Manual test kit \\
\hline Botulinum toxins & & \\
\hline Staphylococcal enterotoxin B & & \\
\hline mykotoxin & & Gation + GCS \\
\hline
\end{tabular}

\section{Characteristics of Identification Levels}

In military operations, it is possible to encounter chemicals typically in:

- CBRN incidents, such as in a hostile chemical attack,

- activities near a passive source, such as chemical industrial infrastructure facilities.

In such situations, the sampling and identification of chemicals are critical to determining the follow-up action or reaction. This may be a purely military event, i.e. operational, but often the event may extend to the international political level. For these reasons, AEP-66 publication defines and describes the levels of chemical identification. The purpose is to ensure that the relevant military forces and resources fulfill the established framewors of identification and thus support the required military or civilian efforts.

\subsection{Provisional Identification}

A chemical can be considered as provisionally identified if one of the following criteria is met: 
- chromatographic retention data (retention time, retention index) of the chemical, obtained by measurement on two chromatographic columns with different stationary phases, in agreement with the tabulated values,

- chromatographic retention data (retention time, retention index) of the chemical substance, obtained by measurement on a specific detector, in agreement with the tabulated values.

\subsection{Confirmed Identification}

The identification of a chemical warfare agent can be considered confirmed if one of the following criteria is met:

- complete spectrum obtained by one spectrometric technique (mass spectrometry [MS], nuclear magnetic resonance spectroscopy [NMR], or infrared spectroscopy [IR]) corresponds to the reference spectra in the database. If no molecular ion is present in the mass spectrum, techniques such as chemical ionization must be performed to confirm the molecular mass of the compound,

- chromatographic retention data obtained for the chemical warfare agent during mass spectrometric analysis by monitoring the selected ion (at least 3 ions) correspond to the valid reference standard. The signal of the 3 selected ions must not differ by more than $10 \%$, with respect to the value of the intensities of the valid reference standard. Measurements must be performed under the same experimental conditions. The ions should have coincident maxima, the same peak width at half height, and exhibit a signal-to-noise ratio greater than 3 .

\subsection{Unambiguous Identification}

This level applies to reference stationary laboratories and will not be discussed. To meet it, it is necessary to perform the measurement by two spectrometric techniques and the results must agree with the measurement performed under identical conditions with the valid reference standard.

The above requirements are the basic criterion for DLAB-CHEM. Other important requirements include the need for the presence of a measuring device for basic detection of ionizing radiation; or the availability of defined requirements for the results of analyzes, which exclude the use of certain analytical methods. There are schematic instructions for the preparation of some samples, as well as some reagents that should be used for identification. Moreover, the publication introduces a list of chemicals that a DLAB-CHEM needs to be capable to identify. Technical Means of the ACR Chemical Corps

\section{Technical Means of the ACR Chemical Corps}

\subsection{Deployable Chemical Laboratory AL-2/ch}

The basic technical instrument of the Czech Army chemical laboratories is the Deployable Chemical Laboratory PPCHL-AL-2/ch (AL-2/ch). The laboratory consists of a container mounted on the TATRA T-815 STEELBRO vehicle, and a power plant carried by the Land Rover 130 vehicle. The container laboratory is composed of 3 sections - preparatory, analytical (Fig. 1), and technological. The main task of the laboratory is identification of selected chemical agents, preparation and storage of 
samples for transport to a reference laboratory, and providing information to the commander.

The preparatory section is hermetically separated from the analytical section. In the preparatory section, the lab assistant works with the crude sample and prepares it for the analysis according to the instructions given by the lab chief. For the provisional analysis, the lab assistant is equipped with simple means ( $\mathrm{pH}$ paper, detection paper PP-3 and nerve agent detection paper DETEHIT) and handheld detectors - Raman spectrometer (FirstDefender RM [Thermo Scientific]), and an infrared spectrometer (TruDefender FTX [Thermo Scientific]). In the analytical part, there have been used gas chromatography with mass spectrometry GC/MS (Griffin 465 [FLIR]), Ultraviolet/Visible (UV/VIS) spectrophotometry (Helios $\beta$ [UNICAM]), thin layer chromatography (TLC system CAMAG) and X-ray fluorescence (XRF) spectrometry (ElvaX Mobile [ElvaTech]) methods at present. Formerly, there was also a GC with phosphorus-sulfur detector (SRI 6810C system) and liquid chromatography (Knauer high performance liquid chromatography [HPLC] system) techniques used for the analysis in Al-2/ch. The laboratory personnel follow their own methodological procedures, drawn on the BlueBook publication [6] or scientific articles [7, 8].

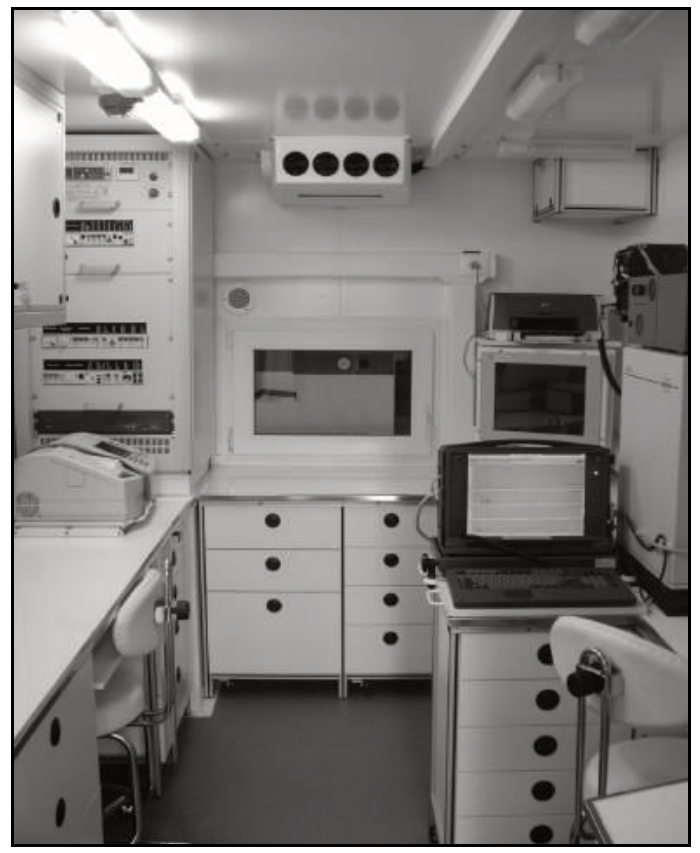

Fig. 1 Analytical section of the AL-2/ch

\subsection{Deployable Chemical Laboratory AL-3/ch}

Soon, the Chemical Corps will fully take advantage of the new deployable laboratory PPCHL-AL-3/ch (AL-3/ch). The modern container part (Fig. 2) forms a complex operational facility for the identification system which is composed of tandem GC/MS/MS system (Griffin 460 [FLIR]), tandem LC/MS/MS system (Ultimate 3000 + LTQ XL [Thermo Scientific]), benchtop infrared spectrometer (Nicolet iS5 [Thermo Scientific]) and an UV/VIS spectrophotometer (Evolution 60S [Thermo Scientific]). 
Elemental analysis will be performed using an XRF spectrometer (ElvaX Mobile [ElvaTech]). The two-chromatography system presents a validation of the identification (LC confirms the GC results), moreover, using ion trap systems offering tandem mass spectrometry. The preparatory part was miniaturized to a glove box, which increased the workplace for the lab personnel. The laboratory will be able to identify chemical warfare agents, selected precursors, drugs, and explosives.

Regarding analysis procedures, the lab personnel will follow the methodological procedures [9] developed during AL-3MET project and BlueBook publication, and scientific articles will be also partly followed.

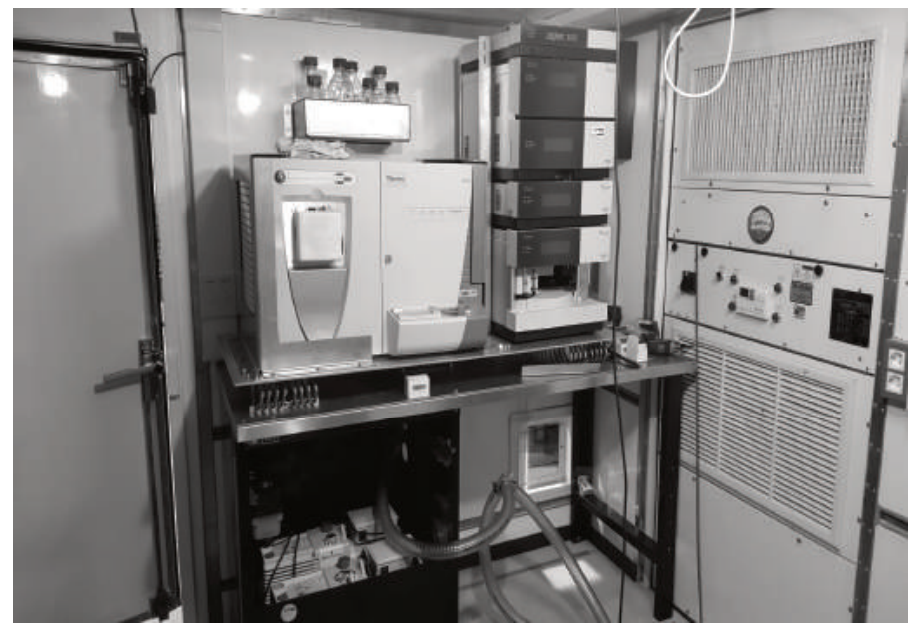

Fig. 2 Internal workplace of the AL-3/ch

\section{Comparative Analysis of the Requirements and Relevant Technical Solutions}

The AL-2/ch laboratory was developed and introduced into Chemical Corps equipment at the beginning of the $21^{\text {st }}$ century. The fact implies that it was not constructed to meet the current requirements. Since then, the laboratory underwent several modernizations in the form of purchase of new devices (new GC/MS/MS system Griffin 465 [FLIR] replaced the old GC/MS EM 640 [Bruker], a XRF spectrometer was purchased, several obsolete equipment has not longer been used - the Knauer HPLC system, GC SRI 6810C, thin layer chromatography), to follow current trends.

The AL-3/ch laboratory was obtained within the framework of the bilateral agreement between the governments of the Czech Republic and USA, and currently it is in the phase of implementation process. In the years 2016-2019, methodological procedures have been developed under the AL-3MET project. The development of the procedures was based on compliance with the requirements of STANAG 4632 and AEP-66 while respecting the actual laboratory equipment and possibilities. The chemical agent identification list was based on the list presented in STANAG 4632 and selected degradation products, precursors, drugs, and explosives were added.

It is appropriate to state that the AEP-66 requirements are not fully met by AL$2 / \mathrm{ch}$ and AL-3/ch laboratories. The following text is focused on the problematic parts of the allied publication in relation to the actual state of the Czech DLAB-CHEM. 


\subsection{Identification of Explosives Capability}

The requirement to identify explosives was fulfilled within the framework of methodological procedures for AL-3/ch development [9]. The creation of agent libraries was based on the analysis of lists of explosives used by the Army of the Czech Republic, and plastic explosives used by the US Army and Russian Army. These sources provided a basis for a list of explosives of interest, and it was followed by the input of spectra and retention index libraries of nitroamines and nitroaromatics to the confirmed identification level.

\subsection{Detection of Ionizing Radiation}

A DLAB-CHEM is not intended to identify ionizing radiation at any identification level. For this purpose, deployable radiological laboratories (DLAB-RAD) are included in the organizational structures of the CBRN Defence battalions. The DLABCHEM only should exclude the presence of an ionizing radiation in the sample by a simple detection technique, or to pass the contaminated sample to a DLAB-RAD, because a radiological sample must not enter the chemical laboratory.

Although detection of ionizing radiation by a DLAB-CHEM is not a preferred requirement in relation to the capability of confirmed level identification achievement, it is a prerequisite prior to analysis start. In the AEP-66, there is a requirement mentioned to implement instructions for the detection of ionizing radiation. Both DLABCHEM follow a system ensuring the passage of the sample contaminated by a radiological agent to the DLAB-RAD in such a way that it does not contaminate the equipment of the chemical laboratory. DLAB-CHEM are not equipped with a radiation detector, however, the presence of a radioactive source is expected to be detected by a sampling team. This team, apart from sampling, performs the provisional detection prior to transport of the sample and the sample sheet to the DLAB. By this measure, contamination of the internal workplace is excluded.

On the second thought, DLAB-CHEM must be capable of sampling according to AEP-66 standards separately. This statement admits that the DLAB-CHEM can operate without the cooperation of a sampling team and should be equipped with the means which will enable to fulfill such tasks.

\subsection{Standard Methods and Raw Data}

In the AEP-66, there is a general statement presented that a DLAB-CHEM must prove the use of methods characterized by accurate and detailed records, and it must be possible to verify the results by tracing the information back to the raw data. In addition, detailed records of service and calibration of all laboratory instruments must be conducted.

Although the requirement is vague, the necessity of software support for all analytical methods used is obvious from the statement. That excludes especially TLC, a valid method according to the methodological procedures of AL-2/ch. TLC is a simple technique based on separation of the sample mixture on a sorbent plate and subsequent coloration change (in case of specific chemical or group of chemicals are present) and subjective visual evaluation of the analysis. This method is also not supported by the identification criteria (see below). Since the UV/VIS spectrophotometry is a questionable method, AEP-66 does not involve this technique. We recommend to 
use the method as a supplementary backup method to get at least certain information about the sample when it is not possible to use any standard methods. UV/VIS spectrophotometry also offers a possibility to provide quantitative information about the sample. Information provided by this method, however, is not supported by AEP-66.

In the case of GC/MS and LC/MS techniques, the requirement is a matter of course and it is possible to trace every command and record realized by these methods.

\subsection{Sample Preparation Techniques}

There are instructions about sample preparation techniques stated in the AEP-66 liquid-liquid extraction, solid phase extraction, solid phase microextraction, hollow fibre liquid phase microextraction, liquid-solid extraction, supercritical fluid extraction, headspace sampling, thermal desorption, derivatization and concentration. These techniques are recommended and it is not necessary to perform all of them, in case that the DLAB-CHEM is able to identify chemical agents using only a selection of those methods.

The AL-2 lab internal area offers a spacious workplace for sample preparation. This fact enables the use of a broad spectrum of equipment like centrifuges, nitrogen flow concentrator, extraction equipment, heaters, ultrasonic cleaner, beakers, vials, pipettes or handheld spectrometers.

The preparatory section was in AL-3/ch reduced to a glove box (Fig. 3), which could represent a quite challenging task for the lab assistant. Planning and coordination aspects are more significant in this type of laboratory. In the AL-3/ch, there is currently not included equipment for some general tasks, and an acquisition will be needed. Recently, a concentrator was purchased, however, a manifold connecting the gas cylinder to the concentrator inside of the glove box would be beneficial. Furthermore, the equipment for solid phase extraction and some other small equipment is missing.

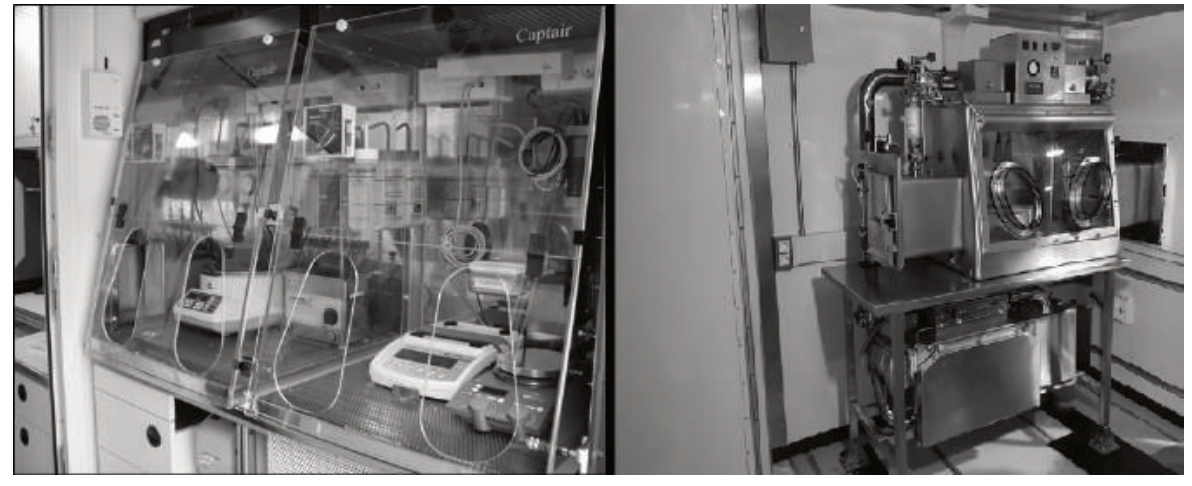

Fig. 3 Preparatory sections in DLAB-CHEM - AL-2/ch (left) and AL-3/ch (right)

In some cases, AEP-66 describes the exact procedural steps for the sample preparation. For example, in solid sample analysis, the sample must be homogenized and divided into four subsamples prior to the analysis of one subsample. From our experience, however, complete homogenization sometimes leads to a fatal impact on the results. A disadvantage of handbooks like AEP-66 is an effort to generalize. In the analysis of chemical warfare agents, the analysts must consider each sample as an individual, and not only follow general procedures. For this purpose, there is an effort 
to engage the chief positions in DLAB-CHEM with professionals with a master or doctoral degree in military chemistry. The procedural steps mentioned above should be considered as recommendations more than a dogma.

\subsection{Chemical Warfare Agents Identification Techniques}

The AEP-66 determines a list of identification techniques for chemical warfare agents. The list states GC, LC, capillary electrophoresis, NMR spectroscopy, infrared spectrometry, and mass spectrometry. Again, it is necessary to mention that some techniques are not supported (TLC, UV/VIS spectrophotometry, Raman spectroscopy, XRF spectrometry). Nevertheless, the last two techniques mentioned are considered to be very helpful. Raman spectroscopy is suitable for the determination of solvent character and for the analysis of solid (powder) samples. The XRF spectrometry will provide important additional information about inorganic samples, and it is a helpful technique when the mass spectrum of an unknown chemical is being interpreted.

These facts represent a disproportion in the AEP-66 requirements. The wellestablished modern instrumental techniques can overlap the allied publication frame, however, it is possible that the results obtained would not be considered as relevant despite the relatively high level of credence or significant support to the results obtained by other techniques.

\subsection{The List of Chemical Agents in AEP-66}

Chemical agents, listed in an appendix of AEP-66, represent a significant problem for meeting the requirements by AL-2/ch and AL-3/ch. Both DLAB-CHEM fulfill this task only partly. The list is basically a transcription of the three Chemical Weapons Convention lists of chemical agents [10]. These lists, however, contain tens of thousands of chemical substances. The AL-3MET project was based on fulfilling the STANAG 4632 requirements. In this document, however, only 18 chemical warfare agents and 5 toxins are mentioned (in case of toxins, a purchase of commercial hand kits). On a second thought, AEP-66 also states that a DLAB-CHEM must fulfill at least STANAG 4632 requirements. According to this statement, AL-3/ch will fulfill the task at the minimal level.

There are two main types of mass analyzers used in field mass spectrometry quadrupole and ion trap. A quadrupole creates mass spectra that are for hundreds of thousands chemicals identical to those saved in available international databases. An unknown chemical substance that will be analyzed by a quadrupole mass spectrometer will provide a spectrum that will respond with a high level of confidence to the spectrum within these databases. An ion trap, on second thought, provides inconsistent spectra dependent on more factors. Due to constructional differences in the ion trap, the chemical spends there more time and this leads to self-protonization, dimerization, and other ballast reactions [11]. The resulting spectrum partly differs from the quadrupole mass spectrum. There are no broad mass spectrum databases measured using ion traps and it is mandatory for the user to create their own database. The purchased Griffin 465 (or 460) GC/MS is an ion trap system. For this reason, it has been necessary to create a user database by analyzing the standards of chemicals of interest. On the other hand, there are some advantages of ion traps, such as MSn tandem spectrometry, which helps to distinguish structural isomers. The ruggedness of the Griffin system enables operation in more demanding field environments when compared to benchtop GC/MS systems. 
Since AL-3/ch introduction, some removable gaps in the laboratory were found. As a possible solution to the problems mentioned above, we recommend completing the equipment with a GC/MS system with a single quadrupole mass analyzer (singlequad GC/MS). This system would work in tandem with Griffin system, and the ion trap GC/MS would be used to fulfill the preliminary identification requirement (it will work with different chromatography column type).

\section{Implications}

\subsection{Fulfillment of Requirements for Provisional Identification}

The general conditions for provisional identification have been described above. It is important to note that their fulfillment should be a prerequisite for a higher level of analysis, i.e. confirmed identification, which can be considered a qualitative superstructure. However, the AL-2/ch laboratory is currently unable to meet the requirements for provisional identification, which already seems to be a problem for achieving a higher level. The first obligation is not fulfilled due to the presence of today's morally and technically obsolete chromatographs - gas chromatograph SRI 6810C and liquid chromatograph Knauer. These instruments have a different column than the currently used Griffin 465 gas chromatograph, but do not meet the mandatory list of substances that the instruments should be able to identify. In addition, it will not be possible to add libraries due to the current technical condition, termination of support, and relatively low sensitivity and resolution. In addition, a standards measurement project would have to be created to supplement libraries with current requirements, which do not balance the benefits obtained by this process. The AEP-66 allows the criterion to be met by the second requirement to obtain chromatographic data identified by a specific detector. Regarding the definitions in IUPAC [12], the only specific detector used in AL-2/ch is the sulfur and phosphorus detector, which is located in the SRI 6810C chromatograph, the problems of which are described above.

Here it is possible to propose the addition of a second GC/MS system with a different column (other than DB-5, which is already involved in GC/MS Griffin 465) in the simplest case. However, this would disrupt a compact design inside the AL-2 container, where "everything has its place". The recent placement / replacement of the essential GC/MS system EM 640 with the Griffin 465 was possible by reducing the work area for the laboratory crew. Problems occur during transport, when the device must be disassembled and placed separately in a special transport package to prevent damage. In the current state, there is no room in the laboratory for another GC/MS system even after its deployment into the field layout. The space can be created by removing the Knauer HPLC and TLC systems, however, the tables in the laboratory only allow the placement of instruments of certain compact dimensions, which most GC/MS systems do not. The problem also occurs with carrier gas distributions, because the current distributions are routed on the opposite side of the container. This would lead to the necessary interventions in the construction of the container.

In the case of the AL-3/ch laboratory, it can be stated that the first criterion is met, namely, the presence of the GC/MS system Griffin 465 with column type DB-5 and LC/MS system Ultimate $3000+$ LTQ XL with column type C18. 


\subsection{Fulfillment of Requirements for Confirmed Identification}

In laboratories AL-2 and AL-3, the first condition of definition is met, but only to a certain extent for a partial list of chemicals (see Tab. 1). In addition, neither the AL$2 / \mathrm{ch}$ nor the AL-3/ch laboratories meet the requirement for the presence of a detector with chemical ionization principle; the absence of a molecular ion can only be expected for lewisites, which are, however, substances in List 1 of the Chemical Weapons Convention. However, it should be noted that this is compensated under DLAB-CHEM methodology using butanethiol derivatization, which reacts with arsenic compounds to form a derivative whose molecular ion is evident in the resulting chromatogram.

The criteria for provisional and confirmed identification again give a clear idea of the techniques that need to be used in today's laboratories, as well as those that will only need to be considered as complementary. It is therefore necessary to state that the established TLC methods and spectrophotometry in laboratories do not provide results that would in any way support specific levels of identification.

\subsection{Summary of Findings}

The comparative analysis of the requirements and the relevant technical solutions pointed to facts that can be considered essential in relation to the ability of the ACR to achieve the Alliance standard. A summary is provided in Tab. 2.

Tab. 2 Fulfillment of AEP-66 requirements by laboratories $A L-2 /$ ch and $A L-3 / c h$

\begin{tabular}{|l|l|l|}
\hline Level of identification & Fulfillment by AL-2/ch & Fulfillment by AL-3/ch \\
\hline \hline Provisional & No & Yes $* *$ \\
\hline Confirmed & Yes $*$ & Yes $* *$ \\
\hline Unambiguous & No $* * *$ & No $* * *$ \\
\hline
\end{tabular}

* Confirmed identification criteria are only achieved at a minimum level. However, due to the nonfulfillment of the requirement of provisional identification, it is not possible to consider them fulfilled, in the sense of achieving a qualitative superstructure.

** The criteria are only met to a minimum for a limited number of chemicals involved. *** Criteria for unambiguous identification are intended for stationary laboratories and are therefore not applicable.

\section{Conclusion}

Both the AL-2/ch and AL-3/ch laboratories have partial shortcomings in meeting the requirements of AEP-66, while the AL-3/ch laboratory will currently meet the requirements for confirmed identification at a minimum level. Problems with meeting the requirements in the case of AL-2/ch are relatively logical given the age of the laboratory. The most problematic is the absence of a quadrupole mass analyzer, which limits the capabilities of laboratories. A defect is also the current absence of a functional two-column system for measuring chromatographic data in the AL-2/ch laboratory, which ultimately does not allow to meet the requirements for specific identification criteria.

Trying to adapt a laboratory to meet the requirements for which it was not designed can be a relatively complex task. The simple acquisition of missing equipment cannot be considered a solution, as it is necessary to respect the relationship between 
the current state, the dimensions of free space, and the location of current equipment in the laboratory. The changes should not only concern the material equipment, but will also require intervention in the construction of the container part. Finally, it should be noted that the article has not yet addressed the need to emphasize the quality and education of staff. The most complex modern equipment needs to meet high requirements for the professional level of crews, the preparation of which cannot be conceived in the form of short-term courses, but somewhat more comprehensively and conceptually.

Analytical chemistry is a field that is evolving at a fast pace. To keep up with the trends, it is necessary to deal with the idea of developing AL-4/ch just in the phase of implementation of the AL-3/ch laboratory. The project of developing a similar device is a time-consuming project, and it can be expected that at the end of such a period, the AL-3/ch hardware will be difficult to compete with the new, modern laboratories of foreign armies. When building a new laboratory, it is recommended to reject the effort to be different, but to emphasize the inspiration obtained on the basis of best practices. Trends in modern analysis of CWAs are obvious and respecting them is the optimal choice to ensure the ability to identify samples at the appropriate level.

\section{Acknowledgement}

The article presents results of the research conducted during a DZRO project "PROTECT" and is largely based on practical testing of military deployable laboratories in field conditions.

\section{References}

[1] V̌sevojsk-2-14. Combat Use of Chemical Troops (in Czech). Prague: Ministry of Defence, 2012.

[2] Bi-SC Capability Codes and Capability Statements [online], 2016. [viewed 202009-09]. Available from: https://fr.scribd.com/document/382349178/CapabilityCodes-and-Capability-Statements-2016-Bi-sc-Nu0083

[3] NATO-STANAG 4632. Deployable NBC Analytical Laboratory. Brussels: NATO Standardization Agency, 2005.

[4] AEP-66, Edition A. NATO Handbook for Sampling and Identification of Biological, Chemical and Radiological Agents (SIBCRA). Brussels: NATO Standardization Office, 2015.

[5] ATP-3.8.1 Volume III. Chemical, Biological, Radiological, and Nuclear Defence Standards for Education, Training and Evaluation. Brussels: NATO Standardization Agency, 2011.

[6] VANNINEN, P. Recommended Operating Procedures for Analysis in the Verification of Chemical Disarmament. Helsinki: University of Helsinki, 2017. ISBN 978-951-51-3916-0.

[7] HAAS, R. Determination of Chemical Warfare Agents: Gas Chromatographic Analysis of Chlorovinylarsines (Lewisite) and their Metabolites by Derivatization with Thiols. Environmental Science and Pollution Research, 1998, 5(1), pp. 2-3. DOI 10.1007/BF02986365.

[8] TERZIC, O., H. GREGG and P. de VOOGT. Identification of Chemicals Relevant to the Chemical Weapons Convention Using the Novel Sample-Preparation 
Methods and Strategies of the Mobile Laboratory of the Organization for the Prohibition of Chemical Weapons. TrAC Trends in Analytical Chemistry, 2015, 65, pp. 151-166. DOI 10.1016/j.trac.2014.10.012.

[9] Preparation of Methodological Procedures and Standard Procedures for Work in Laboratory AL-3 “AL-3MET” (in Czech). Brno: Military Research Institute, 2020.

[10] BOTHE, M. Convention on the Prohibition of the Development, Production, Stockpiling and Use of Chemical Weapons and on their Destruction [online]. Geneva: Audiovisual Library of International Law, 1992. [viewed 2020-10-01]. Available from: https://legal.un.org/avl/ha/cpdpsucw/cpdpsucw.html

[11] VORCE, S.P., J.H. SKLEROV and K.S. KALASINSKY. Assessment of the IonTrap Mass Spectrometer for Routine Qualitative and Quantitative Analysis of Drugs of Abuse Extracted from Urine. Journal of Analytical Toxicology, 2000, 24(7), pp. 595-601. DOI 10.1093/jat/24.7.595.

[12] ETTRE, L.S. Nomenclature for Chromatography (IUPAC Recommendations 1993). Pure and Applied Chemistry, 2009, 65(4), pp. 819-872. DOI 10.1351 /pac199365040819. 